\title{
Un système d'aide au choix de modèles hydrologiques et hydrauliques pour simuler les réseaux d'assainissement : application aux modèles de propagation en conduite A decision support system for the choice of hydrological and hydraulics models in order to simulate urban drainage networks: application on flood routing models
}

\author{
O. Blanpain et B. Chocat
}

Volume 12, numéro 2, 1999

URI : https://id.erudit.org/iderudit/705354ar

DOI : https://doi.org/10.7202/705354ar

\section{Aller au sommaire du numéro}

\section{Éditeur(s)}

Université du Québec - INRS-Eau, Terre et Environnement (INRS-ETE)

\section{ISSN}

0992-7158 (imprimé)

1718-8598 (numérique)

\section{Découvrir la revue}

Citer cet article

Blanpain, O. \& Chocat, B. (1999). Un système d'aide au choix de modèles hydrologiques et hydrauliques pour simuler les réseaux d'assainissement : application aux modèles de propagation en conduite. Revue des sciences de l'eau / Journal of Water Science, 12(2), 317-332. https://doi.org/10.7202/705354ar

\section{Résumé de l'article}

La nouvelle génération de logiciels destinés aux études d'assainissement dispose d'un nombre croissant de modèles hydrauliques et hydrologiques. Il en découle une augmentation des possibilités de choix parmi ces modèles qui complique la tâche des techniciens de l'assainissement. Pour limiter cette difficulté, nous suggérons d'introduire dans les logiciels des outils permettant d'aider les utilisateurs à choisir les modèles en adéquation avec le réseau à simuler. Dans cet article, nous nous intéresserons essentiellement aux modèles de propagation en conduite. Les modèles de propagation les plus usités sont un modèle basé sur les équations de Barré de Saint Venant et des modèles conceptuels nettement plus simples tels que le modèle Muskingum. Ces modèles présentent chacun des avantages et des inconvénients. Dans la pratique, plus un modèle est sophistiqué, mieux il est capable de représenter la réalité. En contrepartie, il est plus difficile à utiliser et nécessite davantage de données et des temps de calcul plus importants. Le problème qui se pose alors à l'utilisateur est de décider quel modèle utiliser. Pour régler ce problème, nous proposons un système d'aide au choix prenant en compte les caractéristiques du réseau étudié, les événements pluvieux simulés et le type d'étude réalisée. Les connaissances nécessaires pour cette aide au choix de modèles peuvent être de qualité variable. Pour mesurer la confiance à accorder à ces connaissances, il est nécessaire de prendre en compte les notions d'imprécision et d'incertitude. Cet état de fait nous a conduit, lors de l'élaboration de cet outil d'aide au choix des modèles de propagation en conduite, à définir un ensemble de règles utilisant la théorie des sous-ensembles flous. 


\title{
Un système d'aide au choix de modèles hydrologiques et hydrauliques pour simuler les réseaux d'assainissement : application aux modèles de propagation en conduite
}

\author{
A decision support system for the choice of hydrological \\ and hydraulics models in order to simulate urban drainage \\ networks: application on flood routing models
}

\section{BLANPAIN ${ }^{1 *}$ et B. CHOCAT ${ }^{2}$}

Reçu le 17 juillet 1997, accepté le 05 septembre 1998**.

\section{SUMMAAY}

Most hydraulic and hydrologic softwares offer an increasing choice of models, each with its advantages and disadvantages. Generally, the more sophisticated the model, the better it can represent larger aspects of reality, but also the more difficult it is to use and the longer the data acquisition and calculation times are. In fact, the real difficulty lies in selecting the appropriate model to use. To answer this question, two subproblems must be solved:

- what is the validity field for each of the models, with respect to the network structure, operating conditions, type of rainfall, nature of problem, etc.?

- once this information is available, what is the best way to ensure its usability, even by people who are not experts in hydrology or hydraulics?

The first problem can be dealt with by analyzing the theoretical validity field of the different models. Nevertheless, this method raises certain difficulties. It requires a particularly thorough knowledge of the equations, algorithms and calculating artifices used in the software package. But, for various reasons, software designers generally refuse to supply this information. Until now, the solution to the second problem has only been addressed by means of scientific reports, communications or papers. The published data generally gives a global introduction to the validity field of each model. Experience shows that most software users do not have enough information on this subject, or, even if they do, do not use it correctly.

To work towards solving these two problems, we propose to introduce into the software packages a decision support system which can help to choose the best model according to the simulated network. In this paper, only the models for

1. Laboratoire Artois Mécanique et Habitat, équipe Méthodes, Université d'Artois, Technoparc Futura, 62400 Béthune, France.

2. URGC Hydrologie Urbaine, INSA de Lyon, Bat 304, 69621 Villeurbanne cedex, France.

- Correspondance.

* Les commentaires seront reçus jusqu'au 30 décembre 1999. 
flow simulation will be taken into account. Presently, the most commonly used models are the more or less complete Barre de Saint Venant equations and more simple conceptual models like Muskingum model.

The major difficulty in solving the first subproblem, was mainly the collection and reformulation of pre-existing knowledge. Considerable bibliographical work had to be supplemented by interviews of experts and by complementary studies (Semsar, 1995) (Mottie, 1996). The result of these studies was the identification of a set of criteria related to the network (slope, fractal dimension, loop index, etc.) or to the working conditions depending on the rainfall event (fullness rate, travel rate, etc.).

The answer to the second problem was to develop an "intelligent" manmachine interface able to analyse the background of the simulation (values of the criteria) and to advise the user on the model to select. The knowledge required to build this decision support system can vary in both source and quality, so assessment of its reliability involve the notions of uncertainty and inaccuracy. The problem of uncertainty has been solved by associating uncertainty degrees to the rules. These degrees define a proposal's level of reliability. The approach to inaccuracy is based on the theory of fuzzy sets, according to which the membership of an element of a given set is not settled but relative. The validity of a given fact is represented by a value between 0 (false) and 1 (true). This value can be related to a variable by fuzzy rules, represented by trapezoidal intervals. By this way, each of the criteria has been represented by qualitative decision variables which allow the elaboration of qualitative rules, leading to a "probably better" decision. One more decision variable - the kind of study - has been added because the hydrograph at the outlet of the network is not necessarily the only criterion to be taken into account. Each of the decision variable is represented by one, two, or sometimes more possible qualitative characterisation(s), associated with a degree of possibility (not probability because the sum of all the possibilities is not necessarily equal to 1 ).

The decision support system uses these variables in a set of rules to determine the degree of adequacy of each model. The development of this system shows us that the use of fuzzy sets and qualitative rules seems to be well adapted to represent the used knowledge. The decision support system will be installed in a software package called CANOE developed by INSA de Lyon and SOGREAH.

In the future we envisage adding an explanatory unit to the decision support system. This study showed too that there is some lack in the knowledge about flow simulation models, so it seems useful to continue to study the validity field of each model.

Key-words: decision support system, flow simulation models, urban storm drainage, fuzzy sets, expert system.

\section{RÉSUMÉ}

La nouvelle génération de logiciels destinés aux études d'assainissement dispose d'un nombre croissant de modèles hydrauliques et hydrologiques. Il en découle une augmentation des possibilités de choix parmi ces modèles qui complique la tâche des techniciens de l'assainissement. Pour limiter cette difficulté, nous suggérons d'introduire dans les logiciels des outils permettant d'aider les utilisateurs à choisir les modèles en adéquation avec le réseau à simuler. Dans cet article, nous nous intéresserons essentiellement aux modèles de propagation en conduite. Les modèles de propagation les plus usités sont un modèle basé sur les équations de Barré de Saint Venant et des modèles conceptuels nettement plus simples tels que le modèle Muskingum. Ces modèles présentent chacun des avantages et des inconvénients. Dans la pratique, plus un modèle 
est sophistiqué, mieux il est capable de représenter la réalité. En contrepartie, il est plus difficile à utiliser et nécessite davantage de données et des temps de calcul plus importants. Le problème qui se pose alors à l'utilisateur est de décider quel modèle utiliser. Pour régler ce problème, nous proposons un système d'aide au choix prenant en compte les caractéristiques du réseau étudié, les événements pluvieux simulés et le type d'étude réalisée. Les connaissances nécessaires pour cette aide au choix de modèles peuvent être de qualité variable. Pour mesurer la confiance à accorder à ces connaissances, il est nécessaire de prendre en compte les notions d'imprécision et d'incertitude. Cet état de fait nous a conduit, lors de l'élaboration de cet outil d'aide au choix des modèles de propagation en conduite, à définir un ensemble de règles utilisant la théorie des sous-ensembles flous.

Mots clés : aide à la décision, modèles de propagation, assainissement pluvial, sous-ensembles flous, système expert.

\section{1 - INTRODUCTION}

Lanalyse du fonctionnement des systèmes d'assainissement urbains par temps de pluie est délicate. Elle nécessite, dans la plupart des cas, l'utilisation de logiciels permettant la simulation des écoulements et des flux de pollution dans le réseau. Les gestionnaires de réseaux d'assainissement ont souvent des difficultés à utiliser de tels logiciels. Ils ne connaissent généralement pas en détail les hypothèses sous-jacentes aux différents modèles utilisés pour représenter les différentes phases du cycle hydrologique, ni leur domaine précis de validité. De ce fait, ils ont du mal à répondre aux questions suivantes : quel modèle choisir ? avec quelles valeurs de paramètres ? quelle confiance accorder aux résultats ? comment améliorer la fiabilité du modèle ou diminuer le temps nécessaire aux calculs ? etc.

La multiplication du nombre et des possibilités des modèles hydrauliques et hydrologiques, prévue pour la génération de logiciels à venir, risque de leur compliquer encore la tâche. Nous avons donc entrepris une recherche sur les moyens de construire une interface homme-machine « intelligente ", c'est-à-dire susceptible d'analyser le contexte (nature du réseau et de l'étude, particularités structurelles et fonctionnelles, problèmes spécifiques, etc.) de façon à conseiller l'utilisateur sur le choix des modèles ou des paramètres. Une telle interface, que nous appellerons " aide au choix de modèle ", sera bientôt disponible sur le logiciel CANOE développé par l'INSA de Lyon et SOGREAH, sur la base de CAREDAS et de CEDRE.

Cet article présente les principes qui seront utilisés dans cette interface en s'appuyant sur une maquette en cours de test à l'URGC Hydrologie urbaine de I'INSA de Lyon. 


\section{2 - OBJECTIFS POURSUIVIS ET LIMITES DU TRAVAIL PRÉSENTÉ}

L'interface d'aide au choix de modèles aura deux fonctions distinctes :

- aider à effectuer un choix parmi une palette de modèles hydrauliques et hydrologiques ;

- aider à déterminer la valeur à donner aux paramètres numériques nécessaires à l'utilisation des modèles choisis.

Ces fonctions sont détaillées dans les paragraphes suivants.

Notre première approche du problème a été une approche experte (BLANPAIN, 1993), (BLANPAIN, CHOCAT, 1994). Cette approche était justifiee par le fait que les connaissances utiles à la mise au point d'un outil de cette nature se prêtaient, dans leur ensemble, assez bien à une formulation sous forme de règles explicites. La difficulté principale a été de réunir et de reformuler un ensemble de connaissances déjà formalisées mais éparses et difficilement utilisables par l'ingénieur ou le technicien, car sous forme de rapports de recherche, de publications scientifiques, de thèses, etc.. II a cependant été nécessaire de compléter ce travail bibliographique :

- par des interviews d'experts, d'une part pour préciser certaines zones "floues ", et d'autre part pour valider l'ensemble des règles ;

- par différentes études permettant de définir les domaines de validité des modèles de propagation en réseau (SEMSAR, 1995) (MOTTIE, 1996) afin de combler un manque de connaissances sur le sujet.

\section{3 - FONCTIONNALITÉS DE L'AIDE AU CHOIX DE MODĖLES}

Les fonctionnalités attendues de l'interface d'aide au choix de modèles sont les suivantes:

a) proposer les modèles hydrauliques ou hydrologiques les mieux adaptés au problème posé (en fonction des résultats escomptés, des données disponibles, des caractéristiques structurelles et fonctionnelles du réseau, de la compatibilité entre les modèles et l'environnement informatique, etc.);

b) valider les modèles choisis par l'utilisateur avant la simulation, c'est-à-dire contrôler a priori la cohérence des choix effectués ;

c) remettre éventuellement en cause les choix précédents après la simulation en fonction des résultats obtenus, c'est-à-dire contrôler a posteriori la bonne utilisation des modèles.

\section{4 - ZONES D'ACTION DE L'AIDE AU CHOIX DE MODĖLES}

Du fait des fonctionnalitės précédemment décrites, l'aide au choix de modèles peut intervenir à différentes étapes du processus de simulation : 
- au début du processus, en fonction du type d'étude et de la zone à étudier, elle propose à l'utilisateur un ensemble de modèles hydrauliques et hydrologiques, correspondant à une description globale de la zone d'étude et aux objectifs poursuivis ;

- l'utilisateur choisit les modèles qu'il souhaite utiliser. Si ces modèles sont différents de ceux proposés, ils sont soumis à un contrôle ;

- après la simulation, les résultats sont analysés et commentés en fonction des modèles utilisés et cette analyse entraîne, s'il y a lieu, une proposition de modification dans le choix des modèles.

\section{5 - LES PHÉNOMĖNES HYDRAULIQUES ET HYDROLOGIQUES RETENUS}

Les phénomènes pris en compte par l'aide au choix de modèles sont :

- les pluies;

- les phénomènes de pertes au ruissellement (production);

- les phénomènes de ruissellement (transfert) ;

- la propagation en conduite ;

- le fonctionnement des ouvrages spéciaux.

Dans les chapitres qui suivront, nous ne détaillerons que ce qui conceme les modèles de propagation en conduite car ces derniers ont un caractère universel alors que les modèles de pluie ou de ruissellement sont généralement rattachés à une zone géographique bien déterminée.

\section{6 - STRUCTURE DE L'AIDE AU CHOIX DE MODÈLES}

La plupart des logiciels d'assainissement offre plusieurs modèles d'écoulement en conduite. Généralement, ils utilisent un modèle basé sur les équations de Barré de Saint Venant et un modèle conceptuel nettement plus simple comme le modèle Muskingum. Ces modèles présentent chacun des avantages et des inconvénients (YEN, 1993). Dans la pratique, plus un modèle est sophistiqué, mieux il est capable de représenter la réalité. En contrepartie, il est plus difficile à utiliser et nécessite davantage de données et des temps de calcul plus importants. Le problème qui se pose alors à l'utilisateur est de décider quel modèle utiliser. Pour régler ce problème, nous proposons un système à base de règles prenant en compte les caractéristiques du réseau étudié, les événements pluvieux simulés et le type d'étude réalisée.

Pour illustrer la forme générale des règles utilisées ainsi que la façon de les manipuler, nous présentons dans ce paragraphe la méthode permettant d'aider l'utilisateur à choisir un modèle de propagation en conduites.

Les variables de décision utilisées peuvent se classer en cinq grandes familles : le type de l'étude engagée (résultats attendus), les grandeurs caractéri- 
sant le réseau, les grandeurs caractéristiques de l'événement simulé, le temps de calcul disponible, les données disponibles.

Dans ce qui suit, nous ne développerons que les règles expertes relatives aux trois premiers points, les outils utilisés pour les deux derniers étant moins originaux.

\subsection{Les types d'étude}

Le type d'étude engagée donne des informations importantes concernant le fonctionnement probable du réseau. Par exemple, si l'utilisateur veut effectuer une simulation pour vérifier le bon fonctionnement du réseau qu'il vient de concevoir, nous supposerons que le réseau fonctionnera probablement à surface libre. Au contraire, si l'utilisateur effectue une simulation pour trouver les causes de dysfonctionnements, nous supposerons que le réseau fonctionnera, au moins en partie, en charge. À partir de ces constatations, nous avons classé les différentes études de la manière suivante:

- recherche des causes de dysfonctionnements;

- contrôle des corrections apportées au réseau pour éliminer les dysfonctionnements ;

- contrôle du bon fonctionnement d'un réseau nouvellement conçu ;

- étude du fonctionnement des déversoirs d'orage ;

- étude du fonctionnement d'un bassin de rétention ;

- étude du fonctionnement des pompes des stations de relèvement ;

- calcul des débits arrivant aux stations d'épuration.

\subsection{Caractérisation du réseau}

Il s'agit, ici, de définir des grandeurs capables de caractériser la morphologie d'un réseau. Nous avons retenu quatre grandeurs caractéristiques (SEMSAR, 1995) :

a) La dimension tractale du réseau. Ce concept a déjà été utilisé pour l'étude des réseaux d'assainissement par S. Thibault (THIBAULT, 1987). La dimension fractale d'un réseau est mesurée par le paramètre $D$ dans l'équation $\mathrm{L}=\mathrm{a} \cdot \mathrm{R}^{\mathrm{D}}$, où $\mathrm{L}$ est la longueur totale du réseau tronqué à une distance $\mathrm{R}$ de l'exutoire. La valeur de $\mathrm{D}$ est comprise entre 1 et 2 (figure 1).

b) Le taux de maillage. Ce paramètre est défini comme le rapport entre le nombre de défluences et le nombre de défluences plus confluences :

$$
M=\frac{\text { nombre de défluences }}{\text { nombre de confluences et défluences }}
$$

La valeur de $M$ varie entre 0 (réseau ramifié) et 1 (réseau fortement maillé) (figure 2).

c) La pente générale du réseau. Nous définirons quatre types de réseaux en fonction de leur pente: les réseaux à pente faible (moins de 0,002 ), les réseaux à pente moyenne (pente voisine de 0,006 ), les réseaux à pente forte (plus de 0,01 ) et les réseaux à pente non homogène (par exemple pente forte en amont et faible en aval).

d) La régularité du réseau. Ce paramètre mesure la régularité du réseau en comparant les taux de saturation relatifs de chacun des tronçons, non pas pour un événement donné, mais pour une période de retour donnée (capacité effective 


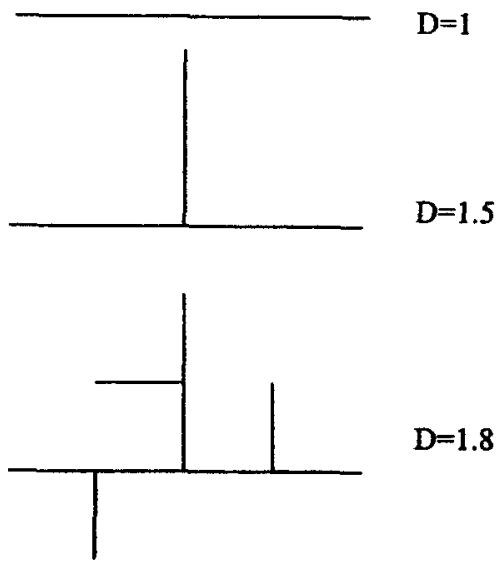

Figure 1 Caractérisation d'un réseau par sa dimension fractale D.

Characterization of a net-work by its fractal dimension $D$.

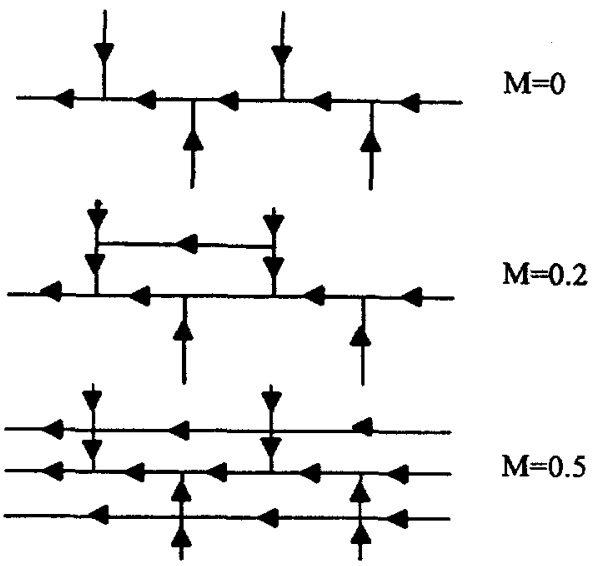

Figure 2 Caractérisation d'un réseau par son taux de maillage $M$.

Characterisation of a network by its loop index.

du tronçon divisée par la capacité d'un tronçon tictif qui assurerait un niveau de protection donné et identique pour tous les tronçons du réseau). Nous pouvons remarquer que de ce fait, elle est donc indépendante de l'événement et intrinsèque au réseau. Le réseau est dit régulier si la valeur de ce rapport est du même ordre de grandeur pour l'ensemble des tronçons. II est dit irrégulier si les valeurs varient d'un tronçon à l'autre.

En remarquant que toutes les possibilités ne sont pas logiques (il est par exemple peu probable d'avoir une forte pente associée à un taux de maillage important ou un taux de maillage important associé à une dimension fractale proche de 1), nous avons retenu 15 classes de réseaux. 


\subsection{Caractérisation de l'événement pluvieux}

De la même manière, nous avons défini deux grandeurs capables de caractériser les événements pluvieux simulés en fonction du réseau étudié :

a) Le taux de remplissage moyen (Tr). Cette grandeur mesure le rapport entre le débit maximum des hydrogrammes et le débit capable des conduites. Pour les réseaux réguliers, la valeur du taux de remplissage est très proche pour l'ensemble des tronçons donc le taux de remplissage moyen est facilement défini. Pour les réseaux irréguliers, nous prendrons le plus grand taux de remplissage comme taux moyen. Une valeur inférieure à $100 \%$ indique que l'écoulement dans le réseau s'effectue à surface libre, une valeur supérieure à $100 \%$ indique un fonctionnement en charge.

b) Le taux de parcours (Tp). Cette grandeur mesure le rapport entre la durée de l'hydrogramme d'entrée et le temps de parcours moyen de l'eau dans le réseau.

À partir de ces deux grandeurs, nous définissons 12 classes d'événements (tableau 1).

Tableau 1 Classification des événements pluvieux.

Table 1 Classification of rainfall.

\begin{tabular}{|c|c|c|c|}
\hline$T p$ & $0,3-0,7$ & $1,5-2,5$ & $>5$ \\
\hline $30-60 \%$ & $\begin{array}{l}\text { intensité de pluie faible } \\
\text { durée de la pluie courte } \\
\text { fonctionnement normal }\end{array}$ & $\begin{array}{l}\text { intensité de pluie faible } \\
\text { durée de la pluie moyenne } \\
\text { fonctionnement normal }\end{array}$ & $\begin{array}{l}\text { intensité de pluie faible } \\
\text { durée de la pluie longue } \\
\text { fonctionnement normal }\end{array}$ \\
\hline $60-100 \%$ & $\begin{array}{l}\text { intensité de pluie moyenne } \\
\text { durée de la pluie courte } \\
\text { limite du fonctionnement } \\
\text { normal }\end{array}$ & $\begin{array}{l}\text { intensité de pluie moyenne } \\
\text { durée de la pluie moyenne } \\
\text { limite du fonctionnement } \\
\text { normal }\end{array}$ & $\begin{array}{l}\text { intensité de pluie moyenne } \\
\text { durée de la pluie longue } \\
\text { limite du fonctionnement } \\
\text { normal }\end{array}$ \\
\hline $100-160 \%$ & $\begin{array}{l}\text { intensité de pluie forte } \\
\text { durée de la pluie courte } \\
\text { dysfonctionnement faible }\end{array}$ & $\begin{array}{l}\text { intensité de pluie forte } \\
\text { durée de la pluie moyenne } \\
\text { dysfonctionnement faible }\end{array}$ & $\begin{array}{l}\text { intensité de pluie forte } \\
\text { durée de la pluie longue } \\
\text { dysfonctionnement faible }\end{array}$ \\
\hline $160-300 \%$ & $\begin{array}{l}\text { intensité de pluie } \\
\text { exceptionnelle } \\
\text { durée de la pluie courte } \\
\text { dysfonctionnement important }\end{array}$ & $\begin{array}{l}\text { intensité de pluie } \\
\text { exceptionnelle } \\
\text { durée de la pluie moyenne } \\
\text { dysfonctionnement important }\end{array}$ & $\begin{array}{l}\text { intensité de pluie } \\
\text { exceptionnelle } \\
\text { durée de la pluie longue } \\
\text { dysfonctionnement impartant }\end{array}$ \\
\hline
\end{tabular}

\subsection{Détermination du domaine d'équivalence des modèles d'écoulement en conduite}

En simulant, les 15 classes de réseaux et les 12 classes d'événements, nous pouvons comparer les résultats fournis par les divers modèles de propagation pour chaque combinaison réseau-événement. Les résultats de cette comparaison ne nous indiqueront pas si un modèle est meilleur qu'un autre (il faudrait pour cela comparer les hydrogrammes obtenus par simulation à des hydrogrammes mesurés sur un réseau réel) ; mais ils nous permettront de savoir dans quels cas les différents modèles de propagation donnent des résultats similaires ou divergents.

Dans le cas de notre étude, nous nous sommes limités à la comparaison des hydrogrammes à l'exutoire du réseau. Nous avons pris comme résultats de réfé- 
rence les hydrogrammes obtenus par un modèle construit à partir du système complet d'équations de Barré de saint Venant. La comparaison s'effectue en comparant les hydrogrammes obtenus par les équations de Barré de Saint Venant (Q1) à ceux obtenus par le modèle étudié (Q2). Pour comparer deux hydrogrammes, nous calculons l'Ecart Quadratique Total (EQT) et le décalage qui minimise EQT entre les deux hydrogrammes discrétisés (SEMSAR, 1995) :

$$
E Q T=\frac{\sqrt{\sum_{i=1}^{n}(Q 1(i \cdot \Delta t)-Q 2(i \cdot \Delta t+\delta))^{2}}}{\sum_{i=1}^{n} Q 1(i \cdot \Delta t)}
$$

Une étude de sensibilité du modèle de Saint Venant (SEMSAR, 1995) a montré que la qualité des résultats pouvait être considérée comme excellente si les deux critères (EQT et $\delta$ ) étaient inférieurs à 0,015 , bonne s'ils étaient compris entre 0,015 et 0,05 et acceptables s'ils étaient inférieurs à 0,1 (figure 3).
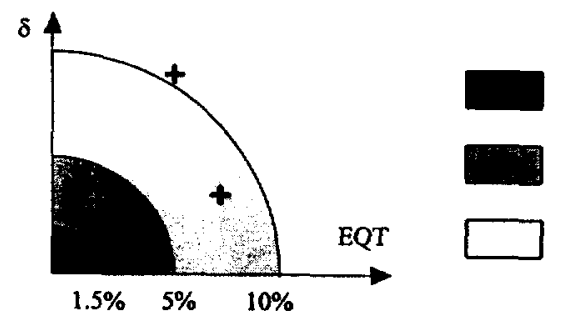

domaine où les résultats sont jugés excellents - domain where the results are assumed to be excellent

domaine où les résultats sont jugés bons - domain where the results are assumed to be good

domaine où les résultats sont jugés acceptables - domain where the results are assumed to be acceptable

Figure 3 Bornes retenues pour qualifier le degré d’équivalence entre les résultats de 'BSV et ceux du modèle à étudier.

Limits retained to qualify the equivalence level between BSV and the studied model results.

Les résultats obtenus sur les 180 cas étudiés (15 réseaux type et 12 événements type) avec deux modèles conceptuels, le modèle Muskingum (SEMSAR, 1995) et le modèle de stock (MOTTIE, 1996), (MOTTIE et al., 1996), fournissent différentes informations. Tout d'abord, ils nous permettent d'une part de connaître les domaines d'équivalence entre ces modèles et le modèle de Barre de Saint Venant. Par exemple pour un réseau fortement maillé avec une faible pente, nous obtenons l'information suivante: Muskingum et Barre de Saint Venant donnent des résultats proches l'un de l'autre (EQT et $\delta$ compris entre 0,015 et 0,05 ) pour une pluie de durée et d'intensité moyennes, alors que pour une pluie courte et de faible intensité, ils donnent des résultats divergents (EQT et $\delta$ supérieurs à 0,1 ). Ils nous permettent également de dégager certaines règles générales concernant l'utilisation des modèles (par exemple : plus un réseau est complexe et plus un événement est long, plus les résultats donnés par les modèles simples se rapprochent des résultats obtenus par le modèle de Saint Venant). Rappelons que ces résultats ne sont valables que lorsqu'il s'agit de connaître le débit à l'exutoire d'un réseau. 


\subsection{La formulation des règles}

\subsubsection{Logique d'ensemble}

La première information traitée par la base de règles est le type d'étude. Selon ce dernier, la méthode d'évaluation des modèles est différente. S'il s'agit de connaître un hydrogramme en un point du réseau (par exemple, les débits arrivant à une station d'épuration, l'hydrogamme d'entrée dans un bassin de rétention, etc.), nous proposons d'utiliser une méthode simplifiée qui s'appuie sur la notion d'équivalence entre modèles développée au 6.4. Cette méthode nous permet de choisir le modèle le plus simple offrant une performance " acceptable ". Dans les autres cas de type d'étude nous utiliserons des règles expertes pour déterminer les conditions d'écoulement probables et en déduire le ou les modèles adéquats.

\subsubsection{Méthode utilisant la notion d'équivalence}

Nous avons déterminé précédemment quinze classes de réseaux (cf. 6.2) et douze classes d'événements pluvieux (ct. 6.3). II s'agit maintenant de déterminer à quelles classes de réseaux et événements, le réseau étudié et l'événement simulé appartiennent. Pour cela, nous avons découpé les domaines de validité des grandeurs caractéristiques en intervalles. Comme il est difficile de déterminer les limites strictes pour chaque intervalle (par exemple, une pente de 0,0035 estelle une pente faible ou moyenne?), nous avons choisi d'utiliser la théorie des sous-ensembles flous (ZADEH, 1965) (ZADEH, 1979). Dans cette théorie, l'appartenance d'une valeur à un intervalle peut être relativisée. L'appartenance à un intervalle est représentée par une valeur f comprise entre 0 et 1 ( 0 indique une nonappartenance, 1 une appartenance totale). Nous pouvons représenter ces degrés d'appartenance par des trapèzes. Les figures 4 et 5 montrent le traitement de la pente et du taux de parcours par des intervalles flous de type trapézoidal.

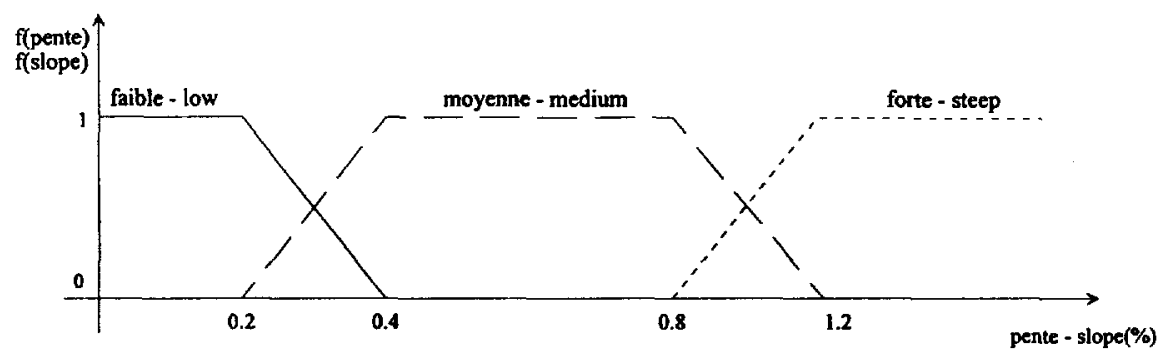

Figure 4 Classification de la pente d'un réseau par sous-ensembles flous.

Classification of a network slope by fuzzy sets.

Les six caractéristiques d'un réseau et d'un événement peuvent être représentées de la sorte. Dès lors, divers degrés d'appartenance aux sous-ensembles flous définis précédemment peuvent être associés au réseau étudié et à l'événement simulé. II ne reste plus qu'à déterminer, à partir de ces degrés d'appartenance, à quelles classes de réseau et d'événement doivent être rattachés le réseau et l'événement étudiés. Pour cela nous proposons la méthode suivante : 


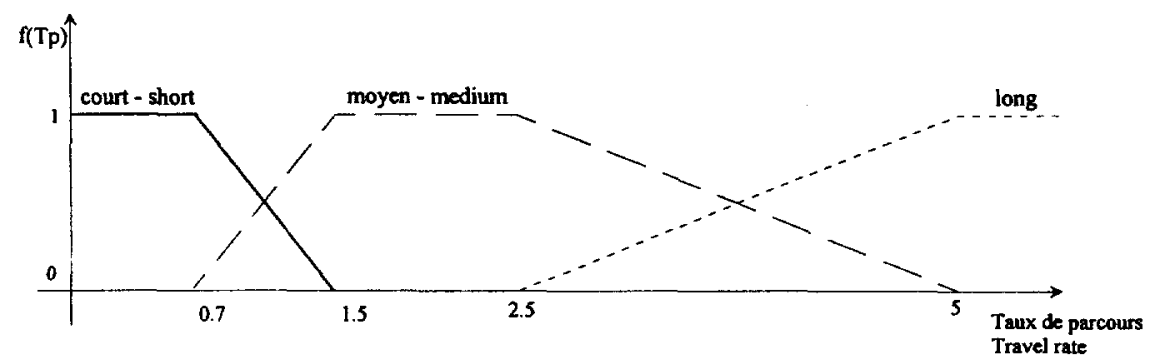

Figure 5 Classification du taux de parcours par sous-ensembles flous.

Classification of the travel rate by fuzzy sets.

Soit $f(i, j)$ le degré d'appartenance de la caractéristique $i(i=1$ à 6$)$ à un intervalle $j$.

Chaque combinaison d'un réseau type et d'un événement type est représentée par un ensemble de valeurs de $f(i, j)$ égales à 0 ou à 1.

De même, au réseau et à l'événement étudiés correspond un ensemble de valeurs de $f(i, j)$ comprises entre 0 et 1 .

\section{a) Première étape : élimination des combinaisons trop différentes}

Pour chaque combinaison type, nous comparons les valeurs de $f(i, j)$ correspondant à la combinaison étudiée (notés $f_{e t}(i, j)$ ) avec les valeurs de $f(i, j)$ correspondant aux combinaisons type (notés $f_{\text {typ }}(i, j)$ ). Si il existe au moins un couple $i, j$ tel que $f_{e t}(i, j)=0$ et $f_{t y p}(i, j)=1$ alors la combinaison étudiée n'est pas assimilable à la combinaison type testée et cette dernière peut être éliminée. De cette manière, nous ne conservons, parmi les 180 combinaisons type, que celles qui sont les plus proches de la combinaison étudiée.

\section{b) Deuxième étape : sélection de la combinaison la plus proche}

Pour chaque combinaison type retenue $k$, nous calculons, pour tout $i$ et $j$ tel que $f_{\text {typ }}(i, j)=1, E(k)=\min \left(f_{e t}(i, j)\right)$.

La combinaison type à laquelle sera rattachée la combinaison étudiée sera celle qui correspondra à $E(k)$ maximum.

Pour mieux comprendre cette méthode, utilisons un exemple. Soient trois caractéristiques $i$ auxquelles sont associés trois sous-ensembles flous j. Les 27 combinaisons type sont représentées par les 9-uplets du type suivant (tableau 2) :

Tableau 2 Exemple de 9-uplets représentant 3 combinaisons type.

Table 2 Example of 9-uplets representing 3 types of combinations.

\begin{tabular}{|c|c|c|c|}
\hline & \multicolumn{3}{|c|}{ combinaison 1} \\
\hline i i & 1 & 2 & 3 \\
\hline 1 & 1 & 0 & 0 \\
\hline 2 & 1 & 0 & 0 \\
\hline 3 & 1 & 0 & 0 \\
\hline
\end{tabular}

\begin{tabular}{|l|l|l|l|}
\cline { 2 - 4 } \multicolumn{1}{c|}{} & \multicolumn{3}{c|}{ combinaison } \\
\hline$i$ & 1 & 2 & 3 \\
\hline 1 & 0 & 1 & 0 \\
\hline 2 & 1 & 0 & 0 \\
\hline 3 & 0 & 1 & 0 \\
\hline
\end{tabular}

\begin{tabular}{|ll|l|l|l|}
\cline { 2 - 4 } \multicolumn{1}{c|}{} & \multicolumn{3}{|c|}{ combinaison 3 } \\
\hline$i$ & $j$ & 1 & 2 & 3 \\
\hline 1 & 0 & 0 & 1 \\
\hline 2 & 1 & 0 & 0 \\
\hline 3 & 0 & 0 & 1 \\
\hline
\end{tabular}


La combinaison étudiée est représentée elle aussi par un 9-uplet (par exemple, celui ci-dessous) (tableau 3):

Tableau 3 Exemple de 9-uplets représentant la combinaison étudiée.

Table 3 Example of 9-uplets representing the studied combination.

\begin{tabular}{|c|c|c|c|}
\hline & \multicolumn{3}{|c|}{ combinaison étudiée } \\
\hline $\begin{array}{ll}i & i\end{array}$ & 1 & 2 & 3 \\
\hline 1 & 0,2 & 0,8 & 0 \\
\hline 2 & $\overline{0,5}$ & 0,4 & 0 \\
\hline 3 & 0,5 & 0,4 & 0 \\
\hline
\end{tabular}

Si nous comparons le 9-uplet de la combinaison type numéro 3 avec le 9-uplet de la combinaison étudiée, nous remarquons que $\left(f_{t y p}(1,3)=1\right.$ et $\left.f_{e t}(1,3)=0\right)$ et $\left(f_{\text {typ }}(3,3)=1\right.$ et $\left.f_{e t}(3,3)=0\right)$, donc la combinaison étudiée ne peut pas correspondre à la combinaison type 3 .

En revanche, elle peut être rattachée aux combinaisons type 1 et 2.

Pour savoir de laquelle de ces dernières elle est la plus proche, nous calculons $E(1)$ et $E(2): E(1)=0,2$ et $E(2)=0,4$.

La valeur de $E$ est maximum pour la seconde combinaison, c'est donc de cette dernière que la combinaison étudiée est la plus proche.

Une fois déterminée la combinaison de type de réseau et d'événement à laquelle nous assimilons la combinaison étudiée, nous comparons les résultats fournis par les différents modèles d'écoulement. Si les résultats sont équivalents nous conseillons le modèle le plus simple. Dans le cas contraire, nous mettons en œuvre la deuxième méthode de sélection.

Remarque : le calcul a priorides valeurs de $f(i, j)$ n'est pas toujours évident. Si la détermination du taux de maillage, de la dimension fractale et de la pente moyenne ne pose pas de problème important, il n'en va pas de même pour celui de la régularité, du taux de remplissage moyen et du taux de parcours. Pour déterminer ces derniers, nous proposons les méthodes suivantes:

- détermination de la régularité : soit l'utilisateur juge par lui-même la régularité du réseau étudié, soit la régularité est estimée suite à une première simulation effectuée avec le modèle le plus rapide et un événement de faible intensité ;

- détermination du taux de parcours : il est possible de l'estimer en prenant une vitesse d'écoulement de l'eau égale à la vitesse à $80 \%$ du débit maximum de la conduite. L'estimation sera vérifiée en calculant le taux de parcours exact après la simulation ;

- détermination du taux de remplissage moyen, son appartenance à un intervalle flou peut être défini par des règles dont voici un exemple:

si l'étude est " recherche des causes de dysfonctionnement " alors le type d'écoulement est soit * dysfonctionnement faible ", soit "dysfonctionnement important * et $f(T, 3)=0,4$ et $f(T r, 4)=0,6$,

si l'étude est " contrôle du bon fonctionnement d'un réseau nouvellement conçu " alors le type d'écoulement est "limite du fonctionnement normal " et $f(T r, 2)=1$. 
Si l'utilisateur a une bonne connaissance de son réseau, il peut choisir de fixer lui même les valeurs à donner aux $f(T r, j)$. Enfin, comme la régularité, le taux de remplissage moyen peut être déduit d'une première simulation effectuée avec le modèle le plus simple.

\subsubsection{Méthode utilisant un système de règles}

Cette méthode se conduit en deux étapes.

Première étape : en fonction du type d'étude, de la morphologie du réseau et, le cas échéant, des résultats obtenus suite à des simulations précédentes, le système détermine les conditions d'écoulement probables.

Deuxième étape : en fonction des types d'écoulement probables, il en déduit le ou les modèles appropriés.

Pour illustrer la façon dont ces différentes informations sont traitées, nous allons détailler une partie des règles de détermination des conditions d'écoulement probables.

\subsubsection{Détermination des conditions d'écoulement probables}

Nous considérerons ici que l'écoulement peut être caractérisé par l'un ou l'autre des phénomènes suivants :

- effets dynamiques importants (ed) ;

- influences aval à surface libre (ia) ;

- mises en charge (mec).

Nota: nous ne nous intéresserons ici qu'aux influences aval et aux effets dynamiques dont la non-prise en compte par certains modèles entraîneraient une erreur dans l'estimation des débits.

La méthode utilisée est la suivante :

Chaque fois que le système applique une règle, il modifie la valeur d'un coefficient de probabilité d'apparition de l'un ou l'autre des phénomènes susceptibles de perturber l'écoulement (ed, ia, mec).

Nota: le vocabulaire utilisé dans ce paragraphe (probable, improbable, possible, etc.) fait référence au sens commun et non au sens mathématique de la théorie des probabilités.

À titre d'exemples, nous présentons ici quelques unes des règles utilisant le type d'étude, la morphologie du réseau et le type d'événement simulé.

\section{a) Type d'étude}

si type d'étude = recherche des causes de dysfonctionnements alors présence de mises en charge probable $\left(^{*}\right)(\operatorname{mec}=m e c+1)$;

si type d"étude = contrôle correction dysfonctionnements alors présence de mises en charge improbable $\left(^{*}\right)(\operatorname{mec}=\operatorname{mec}-1)$;

si type d'étude = contrôle du bon fonctionnement d'un réseau nouvellement conçu alors présence de mises en charge improbable $\left({ }^{*}\right)(\operatorname{mec}=m e c-1)$; 


\section{b) Morphologie du réseau}

si la pente est hétérogène alors présence d'effets dynamiques possible $(e d=e d+1)$; si la pente est faible avec un degré de possibilité $f(p)$ alors la possibilité de présence d'influence aval est renforcée (ia $=i a+f(p)$ );

si la pente est forte avec un degré de possibilité $f(p)$ alors le doute quant à la présence d'effets dynamiques et d'influences aval est renforcé (ia = ia - $f(p)$, ed $=e d-f(p)$ );

si les entrées sont régulièrement réparties alors le doute quant à la présence d'effets dynamiques et d'influences aval est renforcé (ia $=i a-1$, ed $=e d-1$ );

si les entrées sont concentrées alors la possibilité de présence d'effets dynamiques et d'influences aval est renforcée $(i a=i a+1, e d=e d+1)$;

si la dimension fractale du réseau est faible avec un degré de possibilité $f(D)$ alors le doute quant à la présence d'influences aval est renforcé (ia $=i a-f(D))$.

\section{c) Type événement simulé}

si événement simulé est court avec un degré de possibilité $f(T p)$ alors la possibilité de présence d'effets dynamiques et d'influences aval est renforcée $(e d=e d+f(T p)$, ia $=i a+f(T p))$;

si événement simulé est long avec un degré de possibilité $f(T p)$ alors le doute quant à la présence d'effets dynamiques et d'influences aval est renforcé $(i a=i a-f(T p)$, ed $=e d-f(T p))$.

Au fur et à mesure de l'utilisation des règles, le système construit un tableau attribuant une note mesurant le risque d'apparition de chaque type de phénomène. Par exemple (tableau 4):

Tableau 4 Notes de risque.

Table 4 Risk grade.

\begin{tabular}{|c|c|c|}
\hline ia & ed & mee \\
\hline$+1,8$ & +1 & $-0,7$ \\
\hline
\end{tabular}

Les notes données sur cet exemple indiquent que, dans ce cas, le modèle à choisir doit fortement prendre en compte le risque d'influences aval, moyennement les effets dynamiques et pas du tout les mises en charge.

\subsubsection{Choix du modèle approprié}

Le tableau 4 est ensuite pris en compte en utilisant des intervalles à frontières floues permettant d'attribuer, pour chaque indicateur, une note d'adéquation comprise entre 0 et 1 aux modèles de propagation (figure 6). Les diagrammes de la figure 6 tiennent compte du fait qu'à modèle équivalent le modèle le plus simple est préféré, la formulation de Barré de Saint Venant pouvant évidemment simuler toutes les conditions pour lesquelles Muskingum s'applique.

En reprenant l'exemple précédent, on obtient ainsi (tableau 5).

Le modèle choisi est celui qui présente la plus forte note minimum Dans notre cas, les minimum sont, pour Muskingum, 0,1 , pour le modèle de stock, 0,5 , pour $\mathrm{BSV}, 0,15$. Le maximum des minimum est 0,5 donc le modèle le plus adéquat, pour la simulation envisagée, est le modèle de stock. 

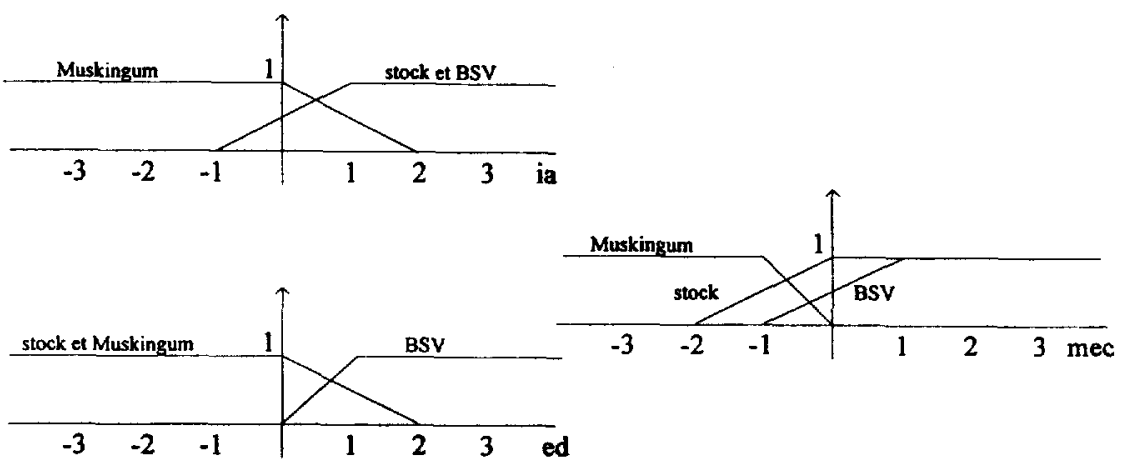

Figure 6 Fonctions d'adéquation des modèles de propagation en conduite.

Appropriateness functions for flow simulation models.

Tableau 5 Degrés d'adéquation pour chaque indicateur.

Table 5 Appropriateness degrees for each indicator.

\begin{tabular}{|lccc|}
\hline & Muskingum & stock & B.S.V. \\
\hline indicateur ia & 0,1 & 1 & 1 \\
indicateur ed & 0,5 & 0,5 & 1 \\
indicateur mec & 0,7 & 0,85 & 0,15 \\
\hline
\end{tabular}

\section{7- CONCLUSION}

Les conclusions que nous pouvons tirer de ce travail sont multiples.

Tout d'abord, il confirme que les connaissances disponibles se prêtent relativement bien à l'élaboration d'un système automatique d'aide à l'utilisation d'un logiciel complexe d'étude des réseaux d'assainissement. Les formalismes proposés (règles de production, utilisation d'intervalles flous et approche symbolique de l'incertain) semblent suffisants pour représenter l'ensemble de l'expertise. Par ailleurs, ils sont faciles à programmer et à introduire dans le logiciel.

La deuxième conclusion concerne l'efficacité opératoire d'un tel outil. Bien que les tests ne soient pas encore terminés, il semble dès à présent qu'une aide disponible en ligne fournisse un réel plus au système en facilitant son utilisation et en évitant de nombreuses erreurs. L'efficacité du système peut être encore renforcée par l'adjonction ultérieure d'un mode explicatif couplé à un module didactique.

Enfin, le travail effectué jusqu'ici en mettant en évidence la diversité des éléments à prendre en compte pour choisir un ensemble de modèles de simulation a permis de déceler les manques existants dans les connaissances relatives à ces modèles. Les études menées par (SEMSAR, 1996) et (MOTTIE, 1996) (CHOCAT et al., 1996) ont permis de combler une partie des lacunes quant aux modèles de propagation en conduite mais elle est encore incomplète. 


\section{RÉFÉRENCES BIBLIOGRAPHIQUES}

BLANPAIN O., 1993. Intégration d'aides expertes dans un logiciel technique d'assainissement. Thèse de Doctorat: INSA de Lyon, France, $239 \mathrm{p}$.

BLANPAIN O., CHOCAT B., 1994. Introduction of expertise in a hydroinformatics system : Choice of hydraulic and hydrologic models. Proceedings of Hydroinformatics 94, Delft, 211-216.

CHOCAT B., SEMSAR A., BLANPAIN 0 ., 1996. Elaboration of assistance methods and tools in the software packages of urban drainage systems for selection of hydraulic models. Proceedings of ICUSD 96, Hannover, 1563-1568.

MOTTIE O., 1996. Modèle de simulation des écoulements en réseau d'assainissement fondé sur le concept de stockage. Thèse de Doctorat : INSA de Lyon, France, $270 \mathrm{p}$.

MOTTIE O., CHOCAT B., BLANPAIN O., 1996. A storage model for the simulation of the hydraulic behaviour of drainage networks. Proceedings of ICUSO 96, Hannover, 641-646.

SEMSAR A., 1995. Mise au point d'une méthodologie de comparaison des modèles de simulation hydraulique des réseaux d'assainissement. Thèse de Doctorat: INSA de Lyon, France, $272 \mathrm{p}$.

THIBAULT S., 1987. Modélisation morphofonctionnelle des réseaux d'assainissement urbain à l'aide du concept de dimension tractale. Thèse de Doctorat d'État: INSA de Lyon, France, 305 p.

YEN B.C., 1993. Is hydraulics over-used or under-used in urban drainage. Proceedings of 6th ICUSD, 91-97.

ZADEH L.A., 1965. Fuzzy sets. Information and Control, $n^{\circ} 8,338-353$.

ZADEH L.A., 1979. A theory of approximate reasoning. Machine Intelligence (J.E. Hayes, D. Mitchie, L.I. Mikulich, eds), Elsevier, $n^{\circ}$ 9, 149-194. 